Review Article

\title{
Macrophage Migration Inhibitory Factor as an Emerging Drug Target to Regulate Antioxidant Response Element System
}

\author{
Hiroshi Yukitake, Masayuki Takizawa, and Haruhide Kimura \\ Pharmaceutical Research Division, Takeda Pharmaceutical Company Ltd., 26-1 Muraoka-Higashi 2-Chome, Fujisawa, \\ Kanagawa 251-8555, Japan \\ Correspondence should be addressed to Haruhide Kimura; haruhide.kimura@takeda.com
}

Received 25 August 2016; Accepted 13 December 2016; Published 16 January 2017

Academic Editor: Ian Copple

Copyright (C) 2017 Hiroshi Yukitake et al. This is an open access article distributed under the Creative Commons Attribution License, which permits unrestricted use, distribution, and reproduction in any medium, provided the original work is properly cited.

Oxidative stress is involved in pathophysiology and pathological conditions of numerous human diseases. Thus, understanding the mechanisms underlying the redox homeostasis in cells and organs is valuable for discovery of therapeutic drugs for oxidative stress-related diseases. Recently, by applying chemical biology approach with an ARE activator, BTZO-1, we found macrophage migration inhibitory factor (MIF) as a new regulator of antioxidant response element- (ARE-) mediated gene transcription. BTZO1 and its active derivatives bound to MIF and protected cells and organs from oxidative insults via ARE activation in animal models with oxidative stress such as ischemia/reperfusion injury, inflammatory bowel diseases, and septic shock. In this review, we briefly highlight key findings in understanding the MIF-ARE system.

\section{Introduction}

Reactive oxygen species (ROS) and reactive nitrogen species (RNS) play a dual role, beneficial and deleterious functions, in living systems $[1,2]$. ROS at low or moderate physiological concentrations mainly work as important signal mediators in multiple systems, including cellular defensive response and induction of a mitogenic response [1]. Excessive level of ROS/ RNS, that is, oxidative stress and nitrosative stress, is harmful and damages cell structures, including lipids and membranes, proteins, and DNA $[1,3,4]$. Overproduction of ROS occurs due to dysregulation of mitochondrial electron transport chain or excessive stimulation of $\mathrm{NAD}(\mathrm{P}) \mathrm{H}$. Oxidative stress and nitrosative stress are associated with imbalance between production of ROS and a function of enzymatic/nonenzymatic antioxidant reaction system in living organisms, and disturbance of this homeostasis plays a critical role in pathophysiology of human diseases, such as cardiovascular diseases, inflammatory bowel diseases, septic shock, rheumatoid arthritis, Alzheimer's disease, Parkinson's disease, multiple sclerosis, amyotrophic lateral sclerosis, schizophrenia, ische$\mathrm{mia} /$ reperfusion, atherosclerosis, diabetes mellitus, cancer, and other diseases, and in aging [1,5-12]. Therefore, regulation of redox homeostasis would be a promising approach in the treatment and/or prevention of these diseases.

Antioxidant response element (ARE) is one of the control batteries for redox homeostasis. Induction of ARE-regulated gene expression is an intrinsic defense system and decreases oxidative stress in cells and organs [13-16]. ARE is a cis-acting DNA regulatory element located in the regulatory regions of multiple genes encoding phase II detoxifying enzymes and cytoprotective proteins, including glutathione S-transferases (GSTs), heme oxygenase-1 (HO-1), reduced nicotinamide adenine dinucleotide phosphate $(\mathrm{NAD}(\mathrm{P}) \mathrm{H})$, quinone oxidoreductases (NQOs), UDP-glucuronosyl transferase (UDPGT), epoxide hydrase, $\gamma$-glutamylcysteine synthetase $(\gamma$ GCS), and peroxiredoxin 1 [13-17]. Thus, activation of the ARE is of critical importance to cellular protection against oxidative stress and could be a therapeutic target for oxidative stress-related diseases. 


\section{Macrophage Migration Inhibitory Factor (MIF) as a Key Regulator of Antioxidant Response Element (ARE) System}

ARE is an enhancer element having the consensus sequence TGACnnnGC $[17,18]$. Many studies have supported the hypothesis that activation of ARE-mediated gene expression is mainly regulated by the transcription factor Nrf2, a member of the cap'n'collar family of basic region-leucine zipper transcription factor [19-22]. Nrf2 is a cytoplasmic protein sequestered by direct binding with the actin-bound protein Keap1 (Kelch ECH associating protein), a Cul3-based E3 ligase [23-25]. Under normal conditions, Nrf2 protein, via direct binding with Keapl, is strictly maintained at low levels by ubiquitination and consequent $26 \mathrm{~S}$ proteasome degradation [23-29]. Under some pathological conditions, oxidative factors dissociate Keap1-Nrf2 complex, and that leads to increase in Nrf2 protein levels and nuclear translocation of $\mathrm{Nrf2}$. Nrf2 in nucleus dimerizes with small Maf proteins and binds to the ARE, resulting in expression of many phase II detoxyfying and cytoprotective genes [30, 31]. Thus, Keap1Nrf2 system is an attractive target for the induction of AREmediated gene expression.

We recently identified macrophage migration inhibitory factor (MIF) as a key regulator of ARE-mediated gene expression by chemical biology approach using BTZO-1, a 1,3benzothiazin-4-on derivative, as chemical probe [32]. MIF, also named as glycosylation-inhibiting factor or phenylpyruvate tautomerase, was originally identified as a soluble factor with macrophage migration-inhibiting properties in the culture medium of activated T lymphocytes [33-37]. MIF has been considered as a cytokine regulating innate and acquired immune responses [37-39]. However, molecular behavior and expression pattern of MIF are different from those of conventional cytokines. For example, MIF is produced by a variety of cells, such as monocytes, macrophages, granulocytes, lymphocytes, eosinophils, neutrophils, endocrine cells, epithelial cells, endothelial cells, and smooth muscle cells, and exists as ubiquitous protein both intra- and extracellular [37, 40-46]. MIF has been reported to have a wide variety of other biological functions, such as counterregulation of glucocorticoid in inflammation, negative regulation of p53-mediated growth arrest and apoptosis, and activation of component of the mitogen-activated protein kinase and Jun-activation domain-binding protein-1 (Jab-1) pathway [47-50]; however, its precise function in the majority of cells is not known.

BTZO-1 was originally discovered from our chemical library as a cardiomyocyte-protective agent; BTZO-1 protected rat primary cardiomyocyte from serum deprivationinduced cell death [32]. Investigation of the mode of action of BTZO-1 revealed that BTZO-1 and its active derivatives activated ARE-mediated gene expression. Drug-affinity chromatography and surface plasmon resonance (SPR) biosensor technique showed that BTZO-1 and its active derivatives in both protection of rat primary cardiomyocyte from serum deprivation-induced cell death and ARE activation directly and selectively bound to MIF [32]. The structure-activity relationship of BTZO-1 derivatives in binding to MIF agreed well with that in ARE-mediated gene expression, as well as cardioprotection. Thus, MIF was considered as a molecular target of BTZO-1. In line with this hypothesis, recombinant purified MIF protein induced ARE-mediated gene expression and suppressed nitric oxide- (NO-) induced cardiomyocyte death in vitro [32]. Furthermore, BTZO-1 promoted MIFinduced ARE activation in $\mathrm{H} 9 \mathrm{c} 2$ cells, while BTZO-1-induced ARE activation was decreased in the MIF-reduced $\mathrm{H} 9 \mathrm{c} 2$ cells transfected with MIF siRNA [32]. The reduction of AREmediated gene expression by BTZO-1 in the MIF-reduced H9c2 cells was restored by applying recombinant MIF to the culture medium. Although the precise intracellular signaling pathway to activate ARE-mediated gene expression after BTZO-1-MIF interaction is unknown, our chemical biological approach with BTZO-1 derivatives suggested that MIF has a pivotal role in ARE-mediated gene regulation [32].

Recently, it was reported that MIF antagonized apoptosis induced by cigarette smoke, a generator of oxidative stress, in human pulmonary endothelial cells, and MIF knockout mice potentiated the toxicity of cigarette smoke exposure via increased apoptosis of endothelial cells [51]. Another study demonstrated that MIF expression levels and cellular antioxidant activity levels were age-dependently decreased in lung, and the analysis with MIF knockout mice revealed that the reductions in MIF expression levels contribute to agerelated radiation-induced lung injury in mice [52], and the decrease in MIF appears to lead to the dysregulation of Nrf2, antioxidant activities, and Nrf2 nuclear concentrations [52]. Moreover, another group demonstrated that MIF provided cardioprotection during ischemia/reperfusion by reducing oxidative stress [53].

These studies support our discovery that MIF works, at least in part, as an upstream signal node for ARE-mediated gene transcription.

\section{Is MIF a Sensor for ARE Activation?}

ARE is also named electrophile/xenobiotic response element, and ARE-mediated gene expression is activated by not only oxidative stress but also electrophilic molecules and heavy metals $[17,25]$. Keap1 is also known as a sensitive sensor not only for oxidative stress but also for xenobiotics such as electrophilic molecules in cytosol [15, 16, 20-28]. Keap1 is a cysteine-rich protein and its cysteine residues have essential roles in Keap1-dependent ubiquitination of Nrf2 [54, 55]. Oxidative/electrophilic molecules modified these cysteine residues, and these modifications resulted in disruption of efficient ubiquitination of Nrf2 and Nrf2 degradation by proteasome. Following the saturation of Keap1 by dysregulation of degradation of Nrf2, newly synthesized Nrf2 proteins accumulate within the cell and translocate to the nucleus, leading to ARE activation $[15,16,20-28,54,55]$.

Our binding assays using both wild type and mutant MIFs demonstrated that BTZO-1-MIF interaction required the intact N-terminal Prol in MIF [32]. Interestingly, Prol is nucleophilic due to its location in a hydrophobic environment and has been reported to interact with electrophiles and alkylating agents [56, 57]. In fact, some of the known ARE 


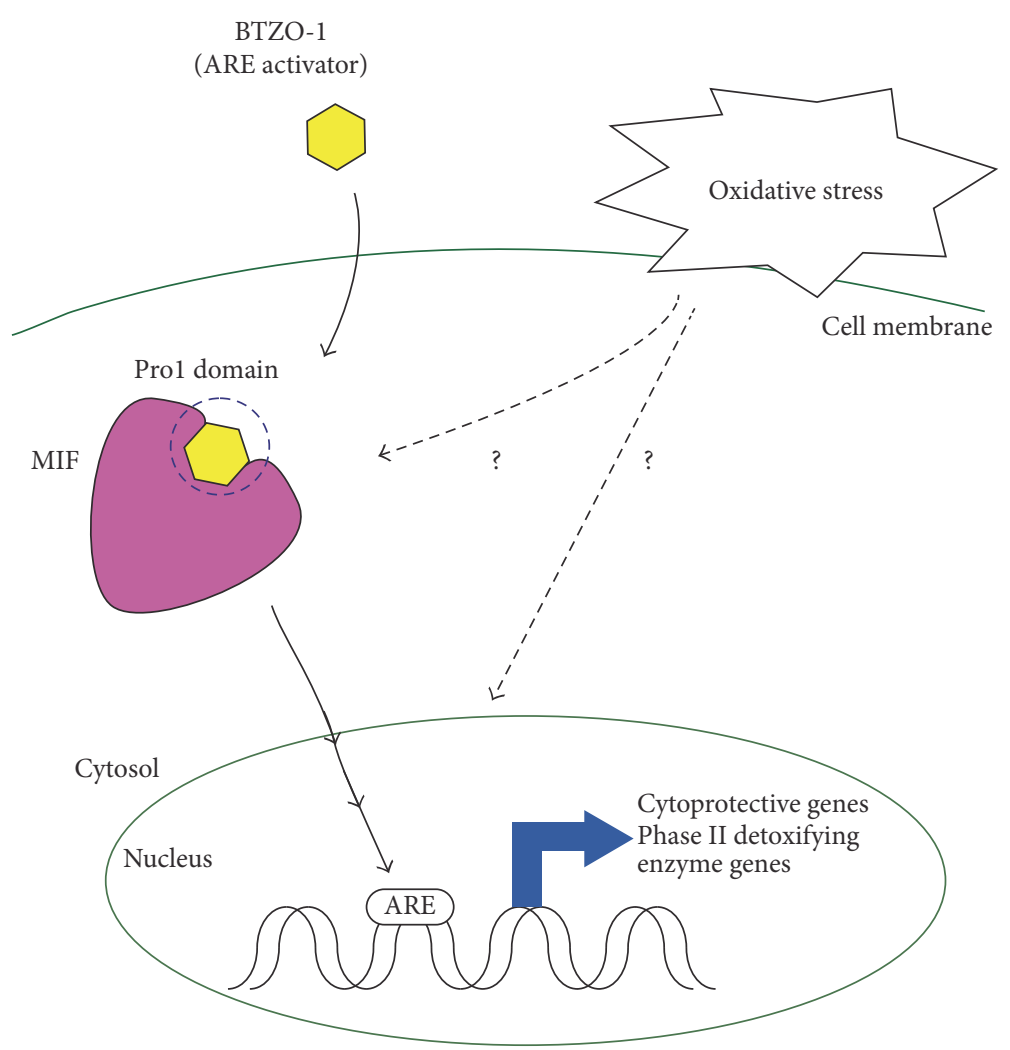

FIGURE 1: BTZO-1 induces ARE-mediated gene expression via MIF under oxidative conditions. MIF has nucleophilic part around N-terminal Prol region and BTZO-1 bound to the Prol region.

activators, such as 15-deoxy- $\Delta 12,14$-prostaglandin J2 (15dPGJ2) a lipid-derived electrophilic molecule, bound to Pro1 site in MIF in the scintillation proximity assay using radiolabeled BTZO-1 as a ligand. Known ARE activators with binding affinity to MIF might activate ARE via binding to MIF [32]. Based on these observations, we propose a hypothesis that MIF N-terminal Prol domain functions as a sensor for deleterious electrophiles. Further studies to investigate this hypothesis are worth trying.

\section{Potential of MIF-ARE System as Therapeutic Target}

Disturbance of redox homeostasis plays a critical role in pathophysiology of several human diseases [1, 5-12]. MIF seems to be a sensor for oxidative stress and/or upstream regulator for ARE as described above; thus, MIF-ARE system may become a new therapeutic target for many diseases caused by excessive oxidative stress. In fact, our recent preclinical studies demonstrated that BTZO-2, a BTZO-1 analog with a better ADME profile, protected heart tissues during ischemia/reperfusion injury in rats [32]. BTZO-2 also protected lipopolysaccharide-induced endotoxic shock in mice [58]. BTZO-15, another active BTZO-1 derivative, ameliorated chemically induced colitis in rats [59]. In addition, 15dPGJ2, which showed MIF binding affinity in a scintillation proximity assay (SPA) [32], exerts anti-inflammatory activity through activation of ARE and suppressed carrageenaninduced pleurisy [60]. Further screenings for ARE activators via MIF are worthwhile, and MIF-ARE system may be a highvalue therapeutic target for wider oxidative stress-related diseases beyond Nrf2-ARE system.

\section{Development of ARE Activators as Therapeutic Drugs}

As discussed, induction of ARE-mediated gene expression is promising as a therapeutic target. However, there are few ARE activators with acceptable safety profiles for therapeutic drugs. ARE system is an intrinsic system against oxidative stress and/or xenobiotics; thus, toxic compounds might show strong efficacy in some drug screening campaigns aiming for ARE-transcriptional activator. These "false-positive toxic compounds" may hinder discovery of safer ARE activators. Therefore, new approaches and/or breakthrough for discovery of safer ARE activators are needed. BTZO-1 was identified in a cell-based and phenotypic screening program aiming for cardioprotective agents. Interestingly, BTZO-1 induced ARE-mediated gene expression without exhibiting cytotoxicity. One unique feature of BTZO-1 is that it activated ARE-mediated gene induction only under oxidative stress conditions, but not under normal conditions (Figure 1) [32]. It is unclear why BTZO-1 induced ARE activation only under 
the oxidative stresses conditions, but this aspect of the unique profile of BTZO-1 could provide a novel avenue for understanding regulation of ARE activation and also for discovery of safer ARE activators. Furthermore, MIF-ARE activators like BTZO-1 may have different pharmacodynamics (PD) profiles compared with Keapl-modifying inducers of Nrf2. The difference in PD profiles will depend on Keapl and MIF expression patterns and their signal contribution in the target tissues for therapy. And if MIF and Keap1 may capture different stresses/ligands, it may also lead to different PD profiles.

\section{Future Directions}

Clinical development of ARE activators as therapeutic drugs is an active area. For example, dimethyl fumarate (DMF) (Tecfidera $^{\mathrm{TM}}$ ), the effects of which are believed, at least in part, to be mediated via Nrf2-ARE system, has been approved by the U.S. Food and Drug Administration as a therapy for multiple sclerosis [61]. CDDO-Me (2-cyano-3,12-dioxooleana-1,9 (11)-diene-28-oic acid methyl ester), also named as bardoxolone methyl, has been clinically studied against a variety of disorders [62]. Although phase III trials of CDDO-Me for chronic kidney disease in the USA failed because of a high rate of cardiovascular adverse events in subpopulation of susceptible patients with an increased risk for heart failure at baseline [63], several clinical trials, such as for treatment of pulmonary hypertension in the US and for chronic kidney disease associated with type 2 diabetes in Japan, are still ongoing. In cancer, the Nrf2-ARE system has emerged as a new therapeutic target [21, 22]. It was reported that Nrf2-ARE system was constitutively activated in some solid tumors, such as lung cancer and esophageal carcinoma, and it contributed to unfavorable prognosis [21, 64-66]. It was traditionally thought that the cancer cells took over and utilized Nrf2-ARE system for survival and malignant growth [21, 64-66]. However, recent reports suggested that Nrf2-ARE activation by CDDO-Me abrogates the immune-suppressive effects of myeloid-derived suppressor cells (MDSCs) and improves immune responses in cancer patients, and Nrf2 activation in MDSCs prevents cancer cell metastasis [22, 6771]. There seems to be room to apply ARE activator to cancer therapy. Some reviews and papers have also pointed out that targeting the Nrf2-ARE system is a promising strategy to tackle neurodegenerative disorders $[16,19,25,72]$.

Here, we propose that MIF could be a critical regulator of ARE-mediated gene expression. Quite interestingly, BTZO-1 activated the ARE-mediated gene expression under oxidative stress conditions without showing cytotoxicity. This unique profile of BTZO-1 may open the door for the discovery of new and safer ARE activators as therapeutic drugs for multiple disorders. Drug screening campaigns using radiolabeled BTZO-1 and MIF protein are worth trying to identify novel MIF binders with potential to activate ARE-mediated transcription. Further studies using BTZO-1 derivatives will be needed to narrow down the best indications for ARE activators using the MIF-ARE system.

\section{Competing Interests}

All authors are employees of Takeda Pharmaceutical Company Limited.

\section{References}

[1] M. Valko, C. J. Rhodes, J. Moncol, M. Izakovic, and M. Mazur, "Free radicals, metals and antioxidants in oxidative stressinduced cancer," Chemico-Biological Interactions, vol. 160, no. 1 , pp. 1-40, 2006.

[2] M. Valko, D. Leibfritz, J. Moncol, M. T. D. Cronin, M. Mazur, and J. Telser, "Free radicals and antioxidants in normal physiological functions and human disease," International Journal of Biochemistry and Cell Biology, vol. 39, no. 1, pp. 44-84, 2007.

[3] W. Dröge, "Free radicals in the physiological control of cell function," Physiological Reviews, vol. 82, no. 1, pp. 47-95, 2002.

[4] K. B. Beckman and B. N. Ames, "The free radical theory of aging matures," Physiological Reviews, vol. 78, no. 2, pp. 547-581, 1998.

[5] I. Dalle-Donne, R. Rossi, R. Colombo, D. Giustarini, and A. Milzani, "Biomarkers of oxidative damage in human disease," Clinical Chemistry, vol. 52, no. 4, pp. 601-623, 2006.

[6] N. S. Dhalla, R. M. Temsah, and T. Netticadan, "Role of oxidative stress in cardiovascular diseases," Journal of Hypertension, vol. 18, no. 6, pp. 655-673, 2000.

[7] P. Jenner, "Oxidative stress in Parkinson's disease," Annals of Neurology, vol. 53, no. S3, pp. S26-S38, 2003.

[8] L. M. Sayre, G. Perry, and M. A. Smith, "Oxidative stress and neurotoxicity," Chemical Research in Toxicology, vol. 21, no. 1, pp. 172-188, 2008.

[9] F. E. Emiliani, T. W. Sedlak, and A. Sawa, "Oxidative stress and schizophrenia: recent breakthroughs from an old story," Current Opinion in Psychiatry, vol. 27, no. 3, pp. 185-190, 2014.

[10] A. Paschos, R. Pandya, W. C. M. Duivenvoorden, and J. H. Pinthus, "Oxidative stress in prostate cancer: changing research concepts towards a novel paradigm for prevention and therapeutics," Prostate Cancer and Prostatic Diseases, vol. 16, no. 3, pp. 217-225, 2013.

[11] M. T. Elnakish, H. H. Hassanain, P. M. Janssen, M. G. Angelos, and M. Khan, "Emerging role of oxidative stress in metabolic syndrome and cardiovascular diseases: important role of Rac/NADPH oxidase," Journal of Pathology, vol. 231, no. 3, pp. 290-300, 2013.

[12] N. A. Simonian and J. T. Coyle, "Oxidative stress in neurodegenerative diseases," Annual Review of Pharmacology and Toxicology, vol. 36, pp. 83-106, 1996.

[13] T. Nguyen, P. J. Sherratt, and C. B. Pickett, "Regulatory mechanisms controlling gene expression mediated by the antioxidant response element," Annual Review of Pharmacology and Toxicology, vol. 43, pp. 233-260, 2003.

[14] X.-L. Chen and C. Kunsch, "Induction of cytoprotective genes through Nrf2/antioxidant response element pathway: a new therapeutic approach for the treatment of inflammatory diseases," Current Pharmaceutical Design, vol. 10, no. 8, pp. 879891, 2004.

[15] J. W. Kaspar, S. K. Niture, and A. K. Jaiswal, "Nrf2:INrf2 (Keap1) signaling in oxidative stress," Free Radical Biology and Medicine, vol. 47, no. 9, pp. 1304-1309, 2009.

[16] H. E. de Vries, M. Witte, D. Hondius et al., "Nrf2-induced antioxidant protection: a promising target to counteract ROSmediated damage in neurodegenerative disease?" Free Radical Biology and Medicine, vol. 45, no. 10, pp. 1375-1383, 2008. 
[17] T. H. Rushmore and C. B. Pickett, "Transcriptional regulation of the rat glutathione S-transferase Ya subunit gene. Characterization of a xenobiotic-responsive element controlling inducible expression by phenolic antioxidants," Journal of Biological Chemistry, vol. 265, no. 24, pp. 14648-14653, 1990.

[18] T. H. Rushmore, M. R. Morton, and C. B. Pickett, "The antioxidant responsive element. Activation by oxidative stress and identification of the DNA consensus sequence required for functional activity," The Journal of Biological Chemistry, vol. 266, no. 18, pp. 11632-11639, 1991.

[19] D. A. Johnson and J. A. Johnson, "Nrf2-a therapeutic target for the treatment of neurodegenerative diseases," Free Radical Biology and Medicine, vol. 88, pp. 253-267, 2015.

[20] Y. Huang, W. Li, Z.-Y. Su, and A.-N. T. Kong, "The complexity of the Nrf2 pathway: beyond the antioxidant response," Journal of Nutritional Biochemistry, vol. 26, no. 12, pp. 1401-1413, 2015.

[21] A. L. Furfaro, N. Traverso, C. Domenicotti et al., "The Nrf2/HO1 axis in cancer cell growth and chemoresistance," Oxidative Medicine and Cellular Longevity, vol. 2016, Article ID 1958174, 14 pages, 2016.

[22] T. Suzuki and M. Yamamoto, "Molecular basis of the Keapl-Nrf2 system," Free Radical Biology and Medicine, vol. 88, pp. 93-100, 2015.

[23] K. Itoh, N. Wakabayashi, Y. Katoh et al., "Keap1 represses nuclear activation of antioxidant responsive elements by $\mathrm{Nrf} 2$ through binding to the amino-terminal Neh2 domain," Genes and Development, vol. 13, no. 1, pp. 76-86, 1999.

[24] L. M. Zipper and R. Timothy Mulcahy, "The Keap1 BTB/POZ dimerization function is required to sequester Nrf2 in cytoplasm," Journal of Biological Chemistry, vol. 277, no. 39, pp. 36544-36552, 2002.

[25] I. Buendia, P. Michalska, E. Navarro, I. Gameiro, J. Egea, and R. León, "Nrf2-ARE pathway: an emerging target against oxidative stress and neuroinflammation in neurodegenerative diseases," Pharmacology and Therapeutics, vol. 157, pp. 84-104, 2016.

[26] K. Itoh, N. Wakabayashi, Y. Katoh, T. Ishii, T. O'Connor, and M. Yamamoto, "Keap1 regulates both cytoplasmic-nuclear shuttling and degradation of Nrf2 in response to electrophiles," Genes to Cells, vol. 8, no. 4, pp. 379-391, 2003.

[27] M. McMahon, K. Itoh, M. Yamamoto, and J. D. Hayes, "Keap1dependent proteasomal degradation of transcription factor Nrf2 contributes to the negative regulation of antioxidant response element-driven gene expression," The Journal of Biological Chemistry, vol. 278, no. 24, pp. 21592-21600, 2003.

[28] Y. Katoh, K. Iida, M.-I. Kang et al., "Evolutionary conserved $\mathrm{N}$-terminal domain of Nrf2 is essential for the Keapl-mediated degradation of the protein by proteasome," Archives of Biochemistry and Biophysics, vol. 433, no. 2, pp. 342-350, 2005.

[29] M. McMahon, N. Thomas, K. Itoh, M. Yamamoto, and J. D. Hayes, "Dimerization of substrate adaptors can facilitate Cullinmediated ubiquitylation of proteins by a 'tethering' mechanism: a two-site interaction model for the Nrf2-Keap1 complex," Journal of Biological Chemistry, vol. 281, no. 34, pp. 24756-24768, 2006.

[30] T. W. Kensler, N. Wakabayashi, and S. Biswal, "Cell survival responses to environmental stresses via the Keap1-Nrf2-ARE pathway," Annual Review of Pharmacology and Toxicology, vol. 47, pp. 89-116, 2007.

[31] G. P. Sykiotis and D. Bohmann, "Stress-activated cap'n'collar transcription factors in aging and human disease," Science Signaling, vol. 3, no. 112, p. re3, 2010.

[32] H. Kimura, Y. Sato, Y. Tajima et al., "BTZO-1, a cardioprotective agent, reveals that macrophage migration inhibitory factor regulates are-mediated gene expression," Chemistry and Biology, vol. 17, no. 12, pp. 1282-1294, 2010.

[33] J. R. David, "Delayed hypersensitivity in vitro: its mediation by cell-free substances formed by lymphoid cell-antigen interaction," Proceedings of the National Academy of Sciences of the United States of America, vol. 56, no. 1, pp. 72-77, 1966.

[34] B. R. Bloom and B. Bennett, "Mechanism of a reaction in vitro associated with delayed-type hypersensitivity," Science, vol. 153, no. 3731, pp. 80-82, 1966.

[35] W. Y. Weiser, P. A. Temple, J. S. Witek-Giannotti, H. G. Remold, S. C. Clark, and J. R. David, "Molecular cloning of a cDNA encoding a human macrophage migration inhibitory factor," Proceedings of the National Academy of Sciences of the United States of America, vol. 86, no. 19, pp. 7522-7526, 1989.

[36] E. Rosengren, R. Bucala, P. Åman et al., "The immunoregulatory mediator macrophage migration inhibitory factor (MIF) catalyzes a tautomerization reaction," Molecular Medicine, vol. 2, no. 1, pp. 143-149, 1996.

[37] T. Calandra and T. Roger, "Macrophage migration inhibitory factor: a regulator of innate immunity," Nature Reviews Immunology, vol. 3, no. 10, pp. 791-800, 2003.

[38] J. Bernhagen, R. Krohn, H. Lue et al., "MIF is a noncognate ligand of CXC chemokine receptors in inflammatory and atherogenic cell recruitment," Nature Medicine, vol. 13, no. 5, pp. 587-596, 2007.

[39] D. Rajasekaran, S. Zierow, M. Syed, R. Bucala, V. Bhandari, and E. J. Lolis, "Targeting distinct tautomerase sites of D-DT and MIF with a single molecule for inhibition of neutrophil lung recruitment," The FASEB Journal, vol. 28, no. 11, pp. 4961-4971, 2014.

[40] J. Nishihira, Y. Koyama, and Y. Mizue, "Identification of macrophage migration inhibitory factor (MIF) in human vascular endothelial cells and its induction by lipopolysaccharide," Cytokine, vol. 10, no. 3, pp. 199-205, 1998.

[41] T. Calandra, J. Bernhagen, R. A. Mitchell, and R. Bucala, "The macrophage is an important and previously unrecognized source of macrophage migration inhibitory factor," Journal of Experimental Medicine, vol. 179, no. 6, pp. 1895-1902, 1994.

[42] A. G. Rossi, C. Haslett, N. Hirani et al., "Human circulating eosinophils secrete macrophage migration inhibitory factor (MIF). Potential role in asthma," The Journal of Clinical Investigation, vol. 101, no. 12, pp. 2869-2874, 1998.

[43] K. Imamura, J. Nishihira, M. Suzuki et al., "Identification and immunohistochemical localization of macrophage migration inhibitory factor in human kidney," Biochemistry and Molecular Biology International, vol. 40, no. 6, pp. 1233-1242, 1996.

[44] A. Daryadel, R. F. Grifone, H.-U. Simon, and S. Yousefi, "Apoptotic neutrophils release macrophage migration inhibitory factor upon stimulation with tumor necrosis factor- $\alpha$," Journal of Biological Chemistry, vol. 281, no. 37, pp. 27653-27661, 2006.

[45] T. Shimizu, "Role of macrophage migration inhibitory factor (MIF) in the skin," Journal of Dermatological Science, vol. 37, no. 2, pp. 65-73, 2005.

[46] L. Verschuren, J. H. N. Lindeman, J. Van Hajo Bockel, H. Abdul-Hussien, T. Kooistra, and R. Kleemann, "Up-regulation and coexpression of MIF and matrix metalloproteinases in human abdominal aortic aneurysms," Antioxidants and Redox Signaling, vol. 7, no. 9-10, pp. 1195-1202, 2005.

[47] T. Calandra, J. Bernhagen, C. N. Metz et al., "MIF as a glucocorticoid-induced modulator of cytokine production," Nature, vol. 377, no. 6544, pp. 68-71, 1995. 
[48] J. D. Hudson, M. A. Shoaibi, R. Maestro, A. Carnero, G. J. Hannon, and D. H. Beach, "A proinflammatory cytokine inhibits p53 tumor suppressor activity," Journal of Experimental Medicine, vol. 190, no. 10, pp. 1375-1382, 1999.

[49] R. A. Mitchell, C. N. Metz, T. Peng, and R. Bucala, "Sustained mitogen-activated protein kinase (MAPK) and cytoplasmic phospholipase A2 activation by macrophage migration inhibitory factor (MIF). Regulatory role in cell proliferation and glucocorticoid action," The Journal of Biological Chemistry, vol. 274, no. 25, pp. 18100-18106, 1999.

[50] R. Kleemann, A. Hausser, G. Geiger et al., "Intracellular action of the cytokine MIF to modulate AP-1 activity and the cell cycle through Jabl," Nature, vol. 408, no. 6809, pp. 211-216, 2000.

[51] J. Fallica, L. Boyer, B. Kim et al., "Macrophage migration inhibitory factor is a novel determinant of cigarette smokeinduced lung damage," American Journal of Respiratory Cell and Molecular Biology, vol. 51, no. 1, pp. 94-103, 2014.

[52] B. Mathew, J. R. Jacobson, J. H. Siegler et al., "Role of migratory inhibition factor in age-related susceptibility to radiation lung injury via NF-E2-related factor-2 and antioxidant regulation," American Journal of Respiratory Cell and Molecular Biology, vol. 49, no. 2, pp. 269-278, 2013.

[53] K. Koga, A. Kenessey, S. R. Powell, C. P. Sison, E. J. Miller, and K. Ojamaa, "Macrophage migration inhibitory factor provides cardioprotection during ischemia/reperfusion by reducing oxidative stress," Antioxidants and Redox Signaling, vol. 14, no. 7, pp. 1191-1202, 2011.

[54] I. M. Copple, "The Keap1-Nrf2 cell defense pathway-a promising therapeutic target?" Advances in Pharmacology, vol. 63, pp. 43-79, 2012.

[55] K. I. Tong, A. Kobayashi, F. Katsuoka, and M. Yamamoto, "Twosite substrate recognition model for the Keap1-Nrf2 system: a hinge and latch mechanism," Biological Chemistry, vol. 387, no. 10-11, pp. 1311-1320, 2006.

[56] P. D. Senter, Y. Al-Abed, C. N. Metz et al., "Inhibition of macrophage migration inhibitory factor (MIF) tautomerase and biological activities by acetaminophen metabolites," Proceedings of the National Academy of Sciences of the United States of America, vol. 99, no. 1, pp. 144-149, 2002.

[57] E. Lolis and R. Bucala, "Macrophage migration inhibitory factor," Expert Opinion on Therapeutic Targets, vol. 7, no. 2, pp. 153-164, 2003.

[58] H. Yukitake, H. Kimura, Y. Tajima et al., "BTZO-2, an antioxidant response element-activator, provides protection against lethal endotoxic shock in mice," European Journal of Pharmacology, vol. 700, no. 1-3, pp. 80-85, 2013.

[59] H. Yukitake, H. Kimura, H. Suzuki et al., "BTZO-15, an AREactivator, ameliorates DSS- and TNBS-induced colitis in rats," PLoS ONE, vol. 6, no. 8, Article ID e23256, 2011.

[60] K. Itoh, M. Mochizuki, Y. Ishii et al., “Transcription factor Nrf2 regulates inflammation by mediating the effect of 15-Deoxy$\Delta^{12,14}$-prostaglandin $\mathrm{J}_{2}$," Molecular and Cellular Biology, vol. 24, no. 1, pp. 36-45, 2004.

[61] M. Stangel and R. A. Linker, "Dimethyl fumarate (BG-12) for the treatment of multiple sclerosis," Expert Review of Clinical Pharmacology, vol. 6, no. 4, pp. 355-362, 2013.

[62] P. E. Pergola, P. Raskin, R. D. Toto et al., "Bardoxolone methyl and kidney function in CKD with type 2 diabetes," New England Journal of Medicine, vol. 365, no. 4, pp. 327-336, 2011.

[63] M. P. Chin, D. Wrolstad, G. L. Bakris et al., "Risk factors for heart failure in patients with type 2 diabetes mellitus and stage
4 chronic kidney disease treated with bardoxolone methyl," Journal of Cardiac Failure, vol. 20, no. 12, pp. 953-958, 2014.

[64] X.-J. Wang, Z. Sun, N. F. Villeneuve et al., "Nrf2 enhances resistance of cancer cells to chemotherapeutic drugs, the dark side of Nrf2," Carcinogenesis, vol. 29, no. 6, pp. 1235-1243, 2008.

[65] H. M. Leinonen, E. Kansanen, P. Pölönen, M. Heinäniemi, and A.-L. Levonen, "Dysregulation of the Keap1-Nrf2 pathway in cancer," Biochemical Society Transactions, vol. 43, pp. 645-649, 2015.

[66] E. J. Moon and A. Giaccia, "Dual roles of NRF2 in tumor prevention and progression: possible implications in cancer treatment," Free Radical Biology and Medicine, vol. 79, pp. 292299, 2015.

[67] D. S. Hong, R. Kurzrock, J. G. Supko et al., "A phase I first-inhuman trial of bardoxolone methyl in patients with advanced solid tumors and lymphomas," Clinical Cancer Research, vol. 18, no. 12, pp. 3396-3406, 2012.

[68] Y.-Y. Wang, Y.-X. Yang, H. Zhe, Z.-X. He, and S.-F. Zhou, "Bardoxolone methyl (CDDO-Me) as a therapeutic agent: an update on its pharmacokinetic and pharmacodynamic properties," Drug Design, Development and Therapy, vol. 8, pp. 20752088, 2014.

[69] S. Nagaraj, J.-I. Youn, H. Weber et al., "Anti-inflammatory triterpenoid blocks immune suppressive function of MDSCs and improves immune response in cancer," Clinical Cancer Research, vol. 16, no. 6, pp. 1812-1823, 2010.

[70] H. Satoh, T. Moriguchi, K. Taguchi et al., "Nrf2-deficiency creates a responsive microenvironment for metastasis to the lung," Carcinogenesis, vol. 31, no. 10, pp. 1833-1843, 2010.

[71] K. Hiramoto, H. Satoh, T. Suzuki et al., "Myeloid lineage-specific deletion of antioxidant system enhances tumor metastasis," Cancer Prevention Research, vol. 7, no. 8, pp. 835-844, 2014.

[72] M. Dumont, E. Wille, N. Y. Calingasan et al., "Triterpenoid CDDO-methylamide improves memory and decreases amyloid plaques in a transgenic mouse model of Alzheimer's disease," Journal of Neurochemistry, vol. 109, no. 2, pp. 502-512, 2009. 


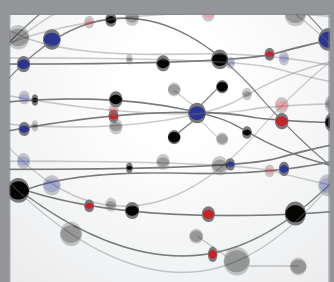

The Scientific World Journal
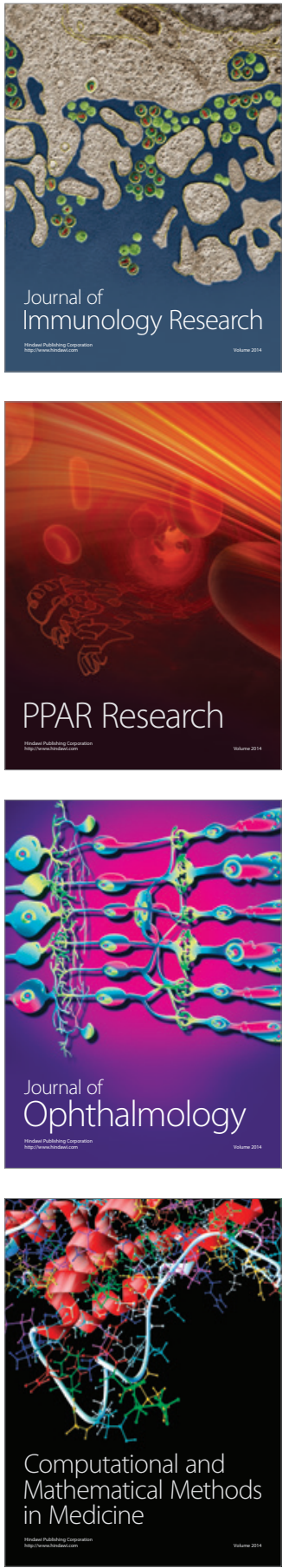

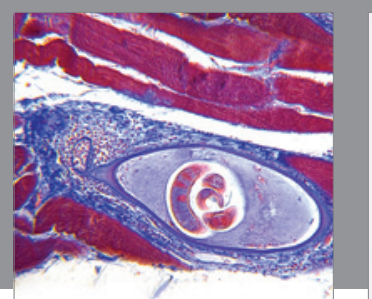

Gastroenterology Research and Practice
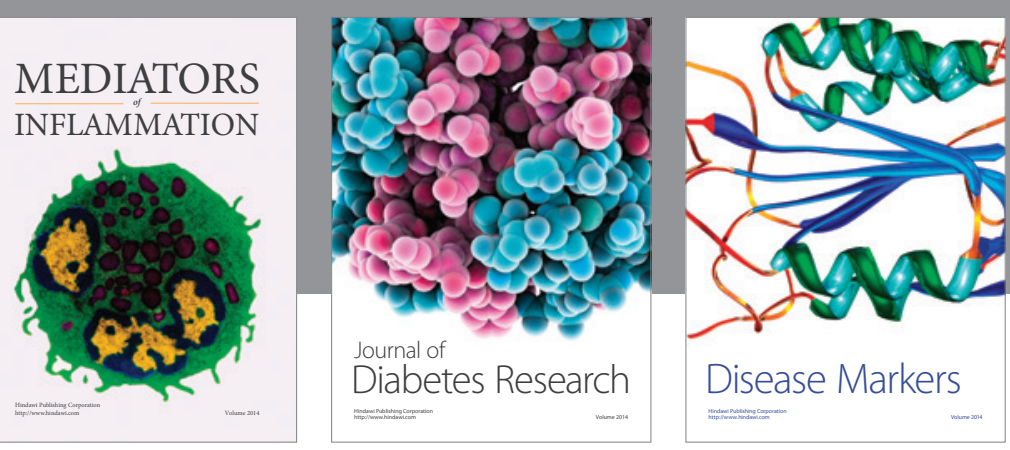

Disease Markers

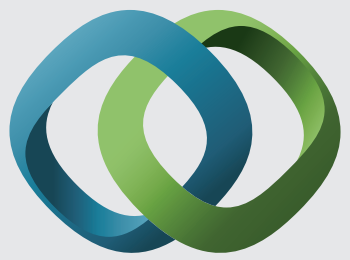

\section{Hindawi}

Submit your manuscripts at

https://www.hindawi.com
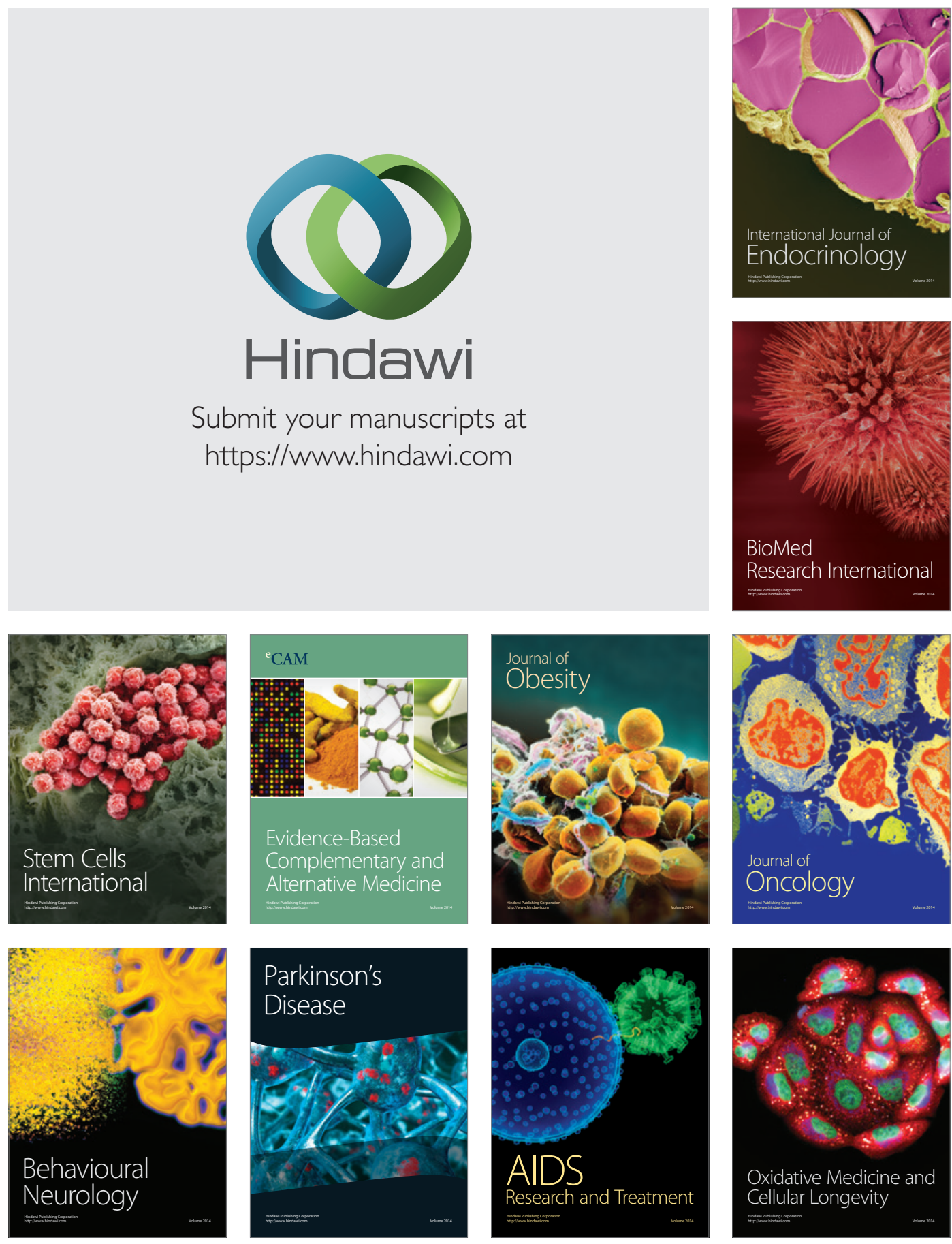